\title{
The structure of the nucleon from generalized parton distributions
}

\section{B. Pasquini*, S. Boffi}

Dipartimento di Fisica Nucleare e Teorica, Università degli Studi di Pavia, and Istituto Nazionale di Fisica Nucleare, Sezione di Pavia, I-27100 Pavia, Italy

E-mail: pasquiniepv.infn.it

\begin{abstract}
Generalized parton distributions (GPDs) have become a standard QCD tool for analyzing and parametrizing the non perturbative parton structure of hadron targets. GPDs might be viewed as non-diagonal overlaps of light-cone wave functions and offer the opportunity to study the partonic content of the nucleon from a new perspective, allowing one to study the interplay between longitudinal and transverse partonic degrees of freedom. In particular, we will review some of the new information encoded in the GPDs through the definition of impact-parameter dependent parton distributions and form factors of the energy-momentum tensor, by exploiting different dynamical models for the nucleon state.
\end{abstract}

LIGHT CONE 2008 Relativistic Nuclear and Particle Physics

July 7-11 2008

Mulhouse, France

${ }^{*}$ Speaker. 


\section{Introduction}

Generalized Parton Distributions (GPDs) have become a standard QCD tool for analyzing and parametrizing the non perturbative parton structure of hadron targets, for reviews see $[1,2,3,4,5]$. GPDs have been introduced in the past in different contexts (see, e.g., [6, 7]), but have raised a large interest in the hadron community only when their importance was stressed in studies of deeply virtual Compton scattering (DVCS) $[8,9,10]$ and hard meson production [11] in connection with the possibility of factorizing their contribution and gaining information on the spin structure of the nucleon [8]. Being defined in terms of nondiagonal matrix elements of the same correlation functions entering the definition of the parton distribution (PDs), GPDs reveal the partonic content of hadrons from a complementary perspective. They do not represent any longer a probability, but rather the interference between amplitudes describing different parton configurations of the nucleon so that they give access to momentum correlations of partons in the nucleon. Furthermore, the finite momentum transfer to the proton makes a second space-time structure of the process possible, and after the Fourier transform in the impact-parameter space allows one to define spin-dependent densities which describe how partons are spatially distributed in the transverse plane [12].

The GPDs can also be viewed as the generating functions for the form factors of the twisttwo operators governing the interaction mechanisms of hard processes in the deep inelastic regime. These generalized form factors do not couple directly to any known fundamental interactions, but can be studied indirectly looking at moments of the GPDs. The most peculiar example are the form factors of the energy-momentum tensor, which give information about the spatial distribution of energy, angular momentum and forces experienced by quarks and gluons inside hadrons.

After summarizing in sect. 2 definition and basic properties of GPDs, in sect. 3 we show results for the GPDs in the impact-parameter space using a light-cone quark model and discussing in particular the correlations of spin and orbital angular momentum of the quarks in the nucleon. Finally, in sect. 4 we review some results for the form factors of the energy-momentum tensor in the framework of the chiral-quark soliton model.

\section{Definition and basic properties of GPDs}

Parton distributions are defined in terms of matrix elements of light-cone bilocal operators between proton states of equal momenta. In general, with initial (final) momentum $p\left(p^{\prime}\right)$ and helicity $\lambda_{N}\left(\lambda_{N}^{\prime}\right)$ one defines a set of quark generalized quark distributions for a hadron with spin $\frac{1}{2}$

$$
\left\langle p^{\prime}, \lambda_{N}^{\prime}\left|\mathscr{O}^{\Gamma}\left(x, \mathbf{0}_{\perp}\right)\right| p, \lambda_{N}\right\rangle,
$$

with

$$
\mathscr{O}^{\Gamma}\left(x, \mathbf{0}_{\perp}\right)=\int \frac{d z^{-}}{4 \pi} e^{i x P^{+} z^{-}} \bar{\psi}_{q}\left(-\frac{z^{-}}{2}, \mathbf{0}_{\perp}\right) \Gamma \psi_{q}\left(\frac{z^{-}}{2}, \mathbf{0}_{\perp}\right) .
$$

In Eq. (2.2) $P=\left(p+p^{\prime}\right) / 2$, and the operator $\Gamma$ is a matrix in Dirac space which selects different spin polarizations of the quark fields. For three particular matrices $\Gamma$ one can classify eight leading twist GPDs: $i$ ) two unpolarized quark GPDs, $H^{q}$ and $E^{q}$, for $\Gamma=\gamma^{+}$; ii) two longitudinally polarized quark GPDs, $\widetilde{H}^{q}$ and $\widetilde{E}^{q}$, for $\Gamma=\gamma^{+} \gamma_{5}$; iii) four quark chiral-odd GPDs for $\Gamma=i \sigma^{i+} \gamma_{5}$, i.e. $H_{T}^{q}$ and $E_{T}^{q}$, which involve the density operator for transversely polarized quarks, and $\widetilde{H}_{T}^{q}$ and 
$\widetilde{E}_{T}^{q}$, defined in terms of a quark operator which flips the transverse spin of the quark. The tilded distributions correspond to matrix elements between nucleon states with flip of the polarization, while the untilded distributions refer to no-flip of the nucleon polarization. Analogous definitions hold for the gluon GPDs.

Because of Lorentz invariance the eight GPDs can only depend on three kinematical variables, i.e. the (average) quark longitudinal momentum fraction $x=k^{+} / P^{+}$, the invariant momentum square $t=\Delta^{2} \equiv\left(p^{\prime}-p\right)^{2}$, and the skewness parameter $\xi$ given by $\xi=-\Delta^{+} /\left(2 P^{+}\right)$. In addition, there is an implicit scale dependence in the definition of GPDs corresponding to the factorization scale $\mu^{2}$ used to separate the (universal) matrix element defining a GPD inside the entire amplitude describing the process under study.

In the forward case, $p=p^{\prime}$, both $\Delta$ and $\xi$ are zero. In this case the functions $H^{q}, \widetilde{H}^{q}$ and $H_{T}^{q}$ reduce to the usual DIS parton distribution functions, i.e. the quark density, helicity and transversity distributions, respectively. No corresponding relations exist for the functions $E^{q}, \widetilde{E}^{q}, E_{T}^{q}$ and $\widetilde{H}_{T}^{q}$, because in the forward limit they decouple in their defining equations. However, they do not vanish. In particular, $E^{q}(x, 0,0)$ carries important information about the quark orbital angular momentum. In contrast, $\widetilde{E}_{T}^{q}(x, 0,0)$ vanishes identically being an odd function of $\xi$ by time reversal symmetry.

Moments in the momentum fraction $x$ play an important role in the theory of GPDs. Weighting Eq. (2.1) with integer powers of $x$ and integrating over $x$, the correlation function $\mathscr{O}^{\Gamma}$ reduces to a local operator and the corresponding matrix elements can be parametrized in terms of generalized form factors (GFFs) related to Mellin moments of the GPDs. More specifically, one has

$$
\int_{-1}^{+1} d x x^{n-1}\left[\begin{array}{c}
H^{q}(x, \xi, t) \\
E^{q}(x, \xi, t)
\end{array}\right]=\sum_{\substack{i=0 \\
\text { even }}}^{n-1}\left[\begin{array}{c}
A_{n, i}^{q}(t) \\
B_{n, i}^{q}(t)
\end{array}\right](2 \xi)^{i} \pm \operatorname{Mod}(n+1,2) C_{n}^{q}(t)(2 \xi)^{n}
$$

for the unpolarized GPDs;

$$
\int_{-1}^{+1} d x x^{n-1} \widetilde{H}^{q}(x, \xi, t)=\sum_{\substack{i=0 \\ \text { even }}}^{n-1}(2 \xi)^{i} \widetilde{A}_{n, i}^{q}(t), \quad \int_{-1}^{+1} d x x^{n-1} \widetilde{E}^{q}(x, \xi, t)=\sum_{\substack{i=0 \\ \text { even }}}^{n-1}(2 \xi)^{i} \widetilde{B}_{n, i}^{q}(t)
$$

for the polarized GPDs;

$$
\begin{aligned}
\int_{-1}^{+1} d x x^{n-1} H_{T}^{q}(x, \xi, t) & =\sum_{\substack{i=0 \\
\text { even }}}^{n-1}(2 \xi)^{i} A_{T n, i}^{q}(t), \quad \int_{-1}^{+1} d x x^{n-1} E_{T}^{q}(x, \xi, t)=\sum_{\substack{i=0 \\
\text { even }}}^{n-1}(2 \xi)^{i} B_{T n, i}^{q}(t),(2.5) \\
\int_{-1}^{1} d x x^{n-1} \widetilde{H}_{T}^{q}(x, \xi, t) & =\sum_{\substack{i=0 \\
\text { even }}}^{n-1}(2 \xi)^{i} \widetilde{A}_{T n, i}^{q}(t), \quad \int_{-1}^{1} d x x^{n-1} \widetilde{E}_{T}^{q}(x, \xi, t)=-\sum_{\substack{i=0 \\
\text { odd }}}^{n-1}(2 \xi)^{i} \widetilde{B}_{T n, i}^{q}(t)
\end{aligned}
$$

for the chiral-odd GPDs.

For the lowest moment $n=1$ in Eqs. (2.3)-(2.4) one finds $A_{1,0}^{q}(t)=F_{1}^{q}(t), B_{1,0}^{q}(t)=F_{2}^{q}(t)$, $\widetilde{A}_{1,0}^{q}(t)=g_{A}^{q}(t)$ and $\widetilde{B}_{1,0}^{q}(t)=g_{P}^{q}(t)$ where $F_{1}^{q}, F_{2}^{q}, g_{A}^{q}$ and $g_{P}^{q}$ are the quark contribution to the Dirac, Pauli, axial and induced pseudoscalar form factors, respectively. Furthermore, in the chiral-odd 
sector one finds $A_{T 1,0}^{q}(t)=g_{T}^{q}(t)$ and $2 \widetilde{A}_{T 1,0}^{1}(0)+B_{T 1,0}^{q}(0)=\kappa_{T}^{q}$, where $g_{T}^{q}$ is the quark tensor form factor and $\kappa_{T}^{q}$ describes how far and in which direction the average position of quarks with spin in the $\hat{x}$-direction is shifted in the $\hat{y}$-direction in an unpolarized nucleon [13].

The second Mellin moments of unpolarized GPDs can be related to the form factors of the energy-momentum tensor (EMT) of QCD by [14]

$$
A_{2,0}^{q}(t)=M_{2}^{q}(t)+\frac{4}{5} d_{1}^{q}(t) \xi^{2}, \quad B_{2,0}^{q}(t)=2 J^{q}(t)-M_{2}^{q}(t)-\frac{4}{5} d_{1}^{q}(t) \xi^{2} .
$$

The form factor $M_{2}(t)$ at $t=0$ reduces to the second Mellin moment of unpolarized parton distributions accessible in inclusive deep inelastic scattering, and represents the fraction of the nucleon momentum carried by quarks. The form factor $d_{1}(t)$ provides information on the distribution of strong forces in the nucleon, similarly as the electromagnetic form factors contain information about the electric charge distribution. The form factor $J^{q}(t)$ is relevant for the spin structure of the nucleon thanks to the so called Ji's sum rule $[8,10]$

$$
J^{q}(t=0)=\left\langle J_{q}^{i}\right\rangle=S^{i}\left[A_{2,0}^{q}(0)+B_{2,0}^{q}(0)\right],
$$

where $\left\langle J_{q}^{i}\right\rangle$ is the total angular momentum along the direction $\hat{i}$ carried by quarks and antiquarks in a proton with spin $S^{i}$. In the case of a proton polarized in the positive $\hat{z}$-direction, one can further split the Ji's sum rules into spin and orbital angular momentum parts, i.e. $\frac{1}{2}=\frac{1}{2} \Sigma^{q}+L^{q}$ where the contribution from the quark spin can be obtained from the moments of the usual polarized quark densities, i.e. $\Sigma^{q}=\widetilde{A}_{1,0}^{q}(t=0)$. The orbital angular momentum has recently been calculated in lattice simulations by the LHPC [15] and QCDSF [16] collaborations. These calculations are for pion masses as low as $350 \mathrm{MeV}$ and volume as large as $(3.5 \mathrm{fm})^{3}$, providing results in the $\overline{\mathrm{M} S}$ renormalization scheme. Extrapolation to the physical pion mass requires a combination of full QCD lattice and Chiral Perturbation Theory [17]. Two remarkable features are found. The first is that the magnitude of the orbital angular momentum contributions of the up and down quarks are separately sizable, $L^{u} \approx-L^{d} \approx 0.30$, yet they cancel nearly completely at all pion masses, $L^{u+d} \approx 0[15,18]$, indicating that the total angular momentum of quarks in the nucleon is of the same size as the quark spin contribution. The second is the close cancellation between the orbital and spin contributions of the down quarks for all pion masses, $J^{d} \approx 0[15,18]$. However, before drawing definite conclusions, one should be aware that these results do not include the contributions from disconnected graphs. Such contributions cancel in the difference of $u$ and $d$ quark distributions but may well be important in their sum.

From the experimental side, first model-dependent constraints on the angular momentum were extracted from recent DVCS data. The analysis was performed comparing data from HERMES [19] on transverse-target and beam-charge asymmetries, and cross section data from JLab [20] with various GPD-models having $J^{q}$ as free parameters [21, 22]. Although the extracted values are strongly model-dependent, such analysis show for the first time that DVCS data have indeed the potential to provide quantitative information about the spin content of the nucleon.

Whereas Eq. (2.8) provides the angular momentum carried by the quarks regardless of their spin, one can also investigate how much each quark polarization component contributes to $\left\langle J_{q}^{i}\right\rangle$. Taking for example the case of transverse polarization in the $\hat{x}$ direction, one can decompose $J_{q}^{x}$ 
with respect to quarks of definite transversity, i.e. $J_{q}^{x}=J_{q,+{ }^{x}}^{x}+J_{q,{ }^{-} x}^{x}$ where $J_{q, \pm{ }^{x}}^{x}$ corresponds to the angular momentum in the $\hat{x}$ direction carried by quarks with transverse polarization in the $\pm \hat{x}$ direction [13]. The transversity components $J_{q, \pm x}^{x}$ can be related to the second Mellin moments of GPDs as follows

$$
\left\langle J_{q, \pm \lambda}^{x}\right\rangle=\frac{S^{x}}{2}\left[A_{2,0}^{q}(0)+B_{2,0}^{q}(0)\right] \pm \frac{1}{4}\left[A_{T 2,0}^{q}(0)+2 \widetilde{A}_{T 2,0}^{q}(0)+B_{T 2,0}^{q}(0)\right] .
$$

In the case of transversely polarized nucleon, $J_{q,+{ }_{x}}^{x}+J_{q,-{ }_{x}}^{x}$ gives the sum rule (2.8), while for unpolarized target only the second term in Eq. (2.9) contributes. Thanks to this decomposition of the angular momentum in transversity components one can gain information on the correlation between the transverse spin and the transverse angular momentum carried by the quarks in an unpolarized target. Note that the same linear combination of GPDs $\left(2 \widetilde{H}_{T}(x, 0,0)+E_{T}(x, 0,0)\right)$ that appears in Eq. (2.9) also describes the transverse displacement of quarks with a given transversity in an unpolarized target relative to the center of momentum (see sect. 3).

\section{Spin densities in the impact-parameter space}

A convenient way to make explicit which kind of information on hadron structure is contained in the GPDs is the representation in terms of overlaps of light-cone wave functions (LCWFs) which are the probability amplitudes to find a given $N$-parton configuration in the Fock-space expansion of the hadron state. In the following, we will confine our analysis to the three-quark sector, by truncating the light-cone expansion of the nucleon state to the minimal Fock-space configuration and adopting a light-cone constituent quark model (CQM) which has been successfully applied in the calculation of the electroweak properties of the nucleon [23].

As outlined in Ref. [24], the starting point is the three-quark wave function obtained as solution of the Schrödinger-like eigenvalue equation in the instant-form dynamics. The corresponding solution in light-cone dynamics is obtained through the unitary transformation represented by product of Melosh rotations acting on the spin of the individual quarks. In particular, the instant-form wave function is constructed as a product of a momentum wave function which is spherically symmetric and invariant under permutations, and a spin-isospin wave function which is uniquely determined by SU(6) symmetry requirements. By applying the Melosh rotations, the Pauli spinors of the quarks in the nucleon rest frame are converted to the light-front spinors. The relativistic spin effects are evident in the presence of spin-flip terms in the Melosh rotations which generate non-zero orbital angular momentum components and non-trivial correlations between spin and transverse momentum of the quarks. On the other hand, the momentum-dependent wave function keeps the original functional form, with instant-form coordinates rewritten in terms of light-front coordinates. Model results for the non-polarized, polarized and chiral-odd GPDs in the momentum space have been presented in Ref. [24, 25], with an extension of the model to include the contribution from higherFock state components in Ref. [25]. Here we focus on the GPDs in the impact-parameter space, which give complementary information to the transverse momentum dependent parton distributions on the spin-spin and spin-orbit correlations of quarks in the nucleon [26].

When $\xi=0$ and $x>0$, by a two-dimensional Fourier transform to impact-parameter space, GPDs can be interpreted as densities of quarks with longitudinal momentum fraction $x$ and transverse location $b$ with respect to the nucleon center of momentum [12, 27]. Depending on the 

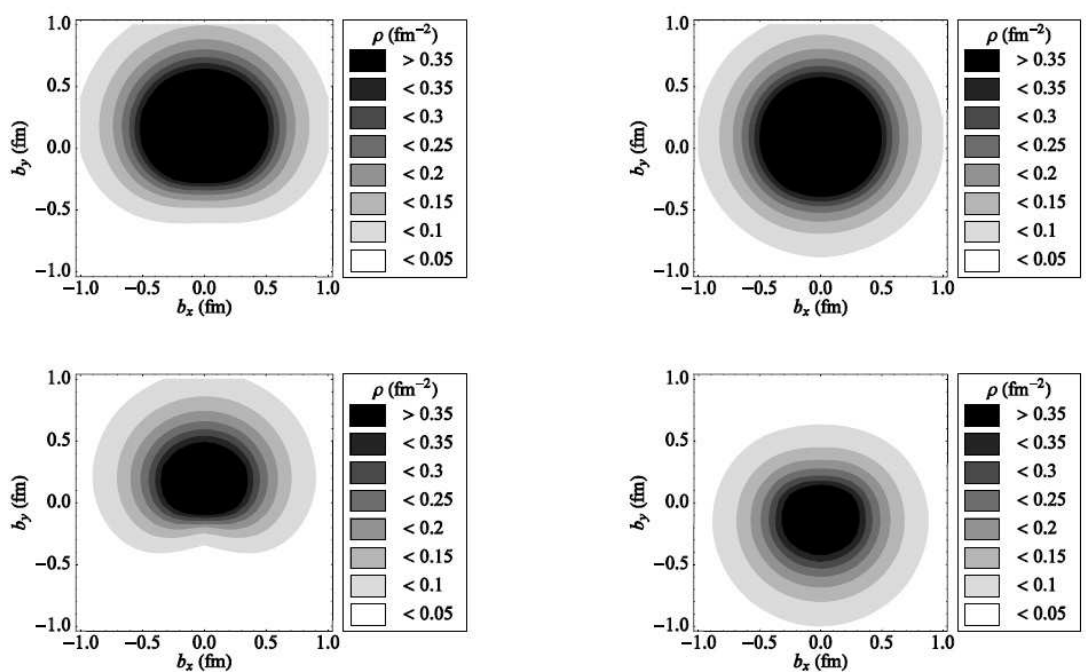

Figure 1: The spin-densities for (transversely) $x$-polarized quarks in an unpolarized proton (left panels) and for unpolarized quarks in a (transversely) $x$-polarized proton. The upper (lower) row corresponds to the results for up (down) quarks.

polarization of both the active quark and the parent nucleon, according to Refs. [27, 28] one defines three-dimensional densities representing the probability to find a quark with transverse spin $s_{T}$ in the nucleon with transverse spin $S_{T}$. It reads

$$
\begin{aligned}
& \rho\left(x, b, s_{T}, S_{T}\right)=\frac{1}{2}\left[H\left(x, b^{2}\right)+s_{T}^{i} S_{T}^{i}\left(H_{T}\left(x, b^{2}\right)-\frac{1}{4 M^{2}} \Delta_{b} \widetilde{H}_{T}\left(x, b^{2}\right)\right)\right. \\
& \left.+\frac{b^{j} \mathcal{E}^{j i}}{M}\left(S_{T}^{i} E^{\prime}\left(x, b^{2}\right)+s_{T}^{i}\left[E_{T}^{\prime}\left(x, b^{2}\right)+2 \widetilde{H}_{T}^{\prime}\left(x, b^{2}\right)\right]\right)+s_{T}^{i}\left(2 b^{i} b^{j}-b^{2} \delta_{i j}\right) S_{T}^{j} \frac{1}{M^{2}} \widetilde{H}_{T}^{\prime \prime}\left(x, b^{2}\right)\right],
\end{aligned}
$$

where the derivatives are defined $f^{\prime}=\frac{\partial}{\partial b^{2}} f$, and $\Delta_{b} f=4 \frac{\partial}{\partial b^{2}}\left(b^{2} \frac{\partial}{\partial b^{2}}\right) f$. In Eq. (3.1) enters the Fourier transform of the GPDs in the impact-parameter space.

In Eq. (3.1), apart from the two orbitally symmetric monopole terms in the first line, there are two dipole structures proportional to $b^{j} \mathcal{E}^{j i} s_{T}^{i}$ and $b^{j} \mathcal{E}^{j i} S_{T}^{i}$, and a quadrupole term proportional to $s_{T}^{i}\left(2 b^{i} b^{j}-b^{2} \delta_{i j}\right) S_{T}^{j}$. The (derivatives of the) GPDs $E\left(x, b^{2}\right), E_{T}\left(x, b^{2}\right)+2 \widetilde{H}_{T}\left(x, b^{2}\right)$ and $\widetilde{H}_{T}\left(x, b^{2}\right)$ thus determine how strongly the orbital symmetry in the transverse plane is distorted by the dipole and quadrupole terms.

Lattice calculations accessing the lowest two $x$-moments of the quark transverse-spin densities have recently been presented in Ref. [29]. Here we show some results in the light-cone CQM for the first $x$-moment of the spin distributions in the cases of transversely polarized quarks in an unpolarized nucleon and unpolarized quarks in a transversely polarized nucleon, referring to [30] for the discussions of more complex spin configurations.

In the case of transversely polarized quarks in an unpolarized proton the dipole contribution $E_{T}^{\prime}\left(x, b^{2}\right)+2 \widetilde{H}_{T}^{\prime}\left(x, b^{2}\right)$ introduces a large distortion perpendicular to both the quark spin and the momentum of the proton, as shown in the left column of Fig. 1. Evidently, quarks in this situation also have a transverse component of orbital angular momentum. This effect has been related [13] 
to a non-vanishing Boer-Mulders function $h_{1}^{\perp}$ which describes the correlation between intrinsic transverse momentum and transverse spin of quarks. Such a distortion reflects the large value of the anomalous tensor magnetic moment $\kappa_{T}$ for both flavors. Here, $\kappa_{T}^{u}=3.98$ and $\kappa_{T}^{d}=2.60$, to be compared with the values $\kappa_{T}^{u} \approx 3.0$ and $\kappa_{T}^{d} \approx 1.9$ of Ref. [29]. Since $\kappa_{T} \sim-h_{1}^{\perp}$, the present results confirm the conjecture that $h_{1}^{\perp}$ is large and negative both for up and down quarks [13].

As also noticed in Refs. [12, 29] the large anomalous magnetic moments $\kappa^{u, d}$ are responsible for the dipole distortion produced in the case of unpolarized quarks in transversely polarized nucleons (right column of Fig. 1). With the present model, $\kappa^{u}=1.86$ and $\kappa^{d}=-1.57$, to be compared with the values $\kappa^{u}=1.673$ and $\kappa^{d}=-2.033$ derived from data. This effect can serve as a dynamical explanation of a non-vanishing Sivers function $f_{1 T}^{\perp}$ which measures the correlation between the intrinsic quark transverse momentum and the transverse nucleon spin. The present results, with the opposite shift of up and down quark spin distributions, imply an opposite sign of $f_{1 T}^{\perp}$ for up and down quarks [31] as confirmed by the recent observation of the HERMES [32] and COMPASS [33] collaborations. The results in Fig. 1 are also in qualitative agreement with lattice calculations [29].

\section{Energy-Momentum Tensor}

The nucleon EMT form factors were studied in lattice QCD [16], chiral perturbation theory [34], and models such as the Skyrme model [35] and chiral quark soliton model ( $\chi$ QSM) [36, 37]. Here we summarize some interesting features investigated within the $\chi$ QSM when looking at the spatial distribution of the EMT form factors $[14,36]$. The $\chi$ QSM provides a field theoretic description of the nucleon in the limit of a large number of colors $N_{c}$, where the nucleon appears as chiral soliton of a static background field. Numerous nucleonic properties have been described in this model, giving predictions in agreement with phenomenology within an accuracy of (10-30)\%. The spatial distribution of the EMT form factors can be obtained from the Fourier transform with respect to $\Delta$ of the matrix element of the static EMT calculated in the Breit frame [14]. The normalized energy density $4 \pi r^{2} \rho_{E}(r) / M_{N}$ is shown in Fig. 2a as a function of $r$ in the chiral limit of a vanishing pion mass and for the physical situation with a pion mass of $140 \mathrm{MeV}$. In the center
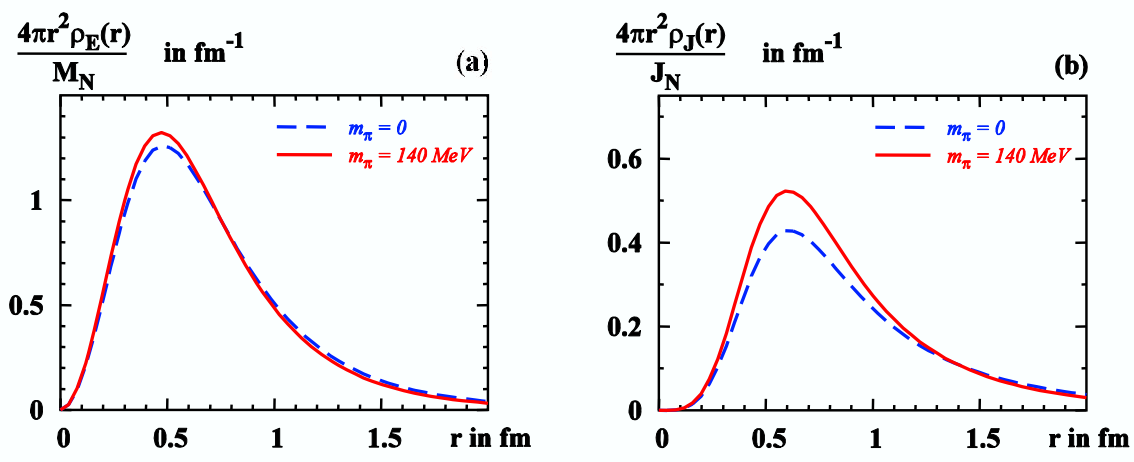

Figure 2: (a) The normalized energy density $4 \pi r^{2} \rho_{E}(r) / M_{N}$ from the $\chi \mathrm{QSM}$ as a function of $r$ in the chiral limit of $m_{\pi}=0$ (dashed curve) and for $m_{\pi}=140 \mathrm{MeV}$ (solid curve). (b) The same for the normalized angular momentum density $4 \pi r^{2} \rho_{J}(r) / J_{N}$ (taken from [36]). 

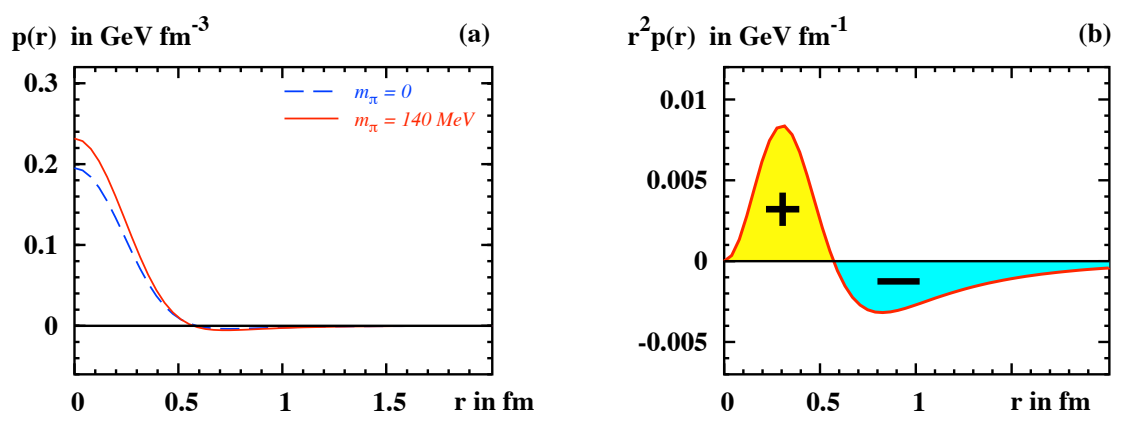

Figure 3: (a) The pressure $p(r)$ from the $\chi \mathrm{QSM}$ as function of $r$ for $m_{\pi}=0$ (dashed curve) and $140 \mathrm{MeV}$ (solid curve). (b) $r^{2} p(r)$ as function of $r$ at the physical value of $m_{\pi}$ (taken from [36]).

of the nucleon the energy density is $\rho_{E}(0)=1.70 \mathrm{GeV} / \mathrm{fm}^{3}$ or $3.0 \times 10^{15} \mathrm{~g} \mathrm{~cm}^{-3}$, corresponding roughly to 13 times the equilibrium density of nuclear matter. As the pion mass decreases, the energy density is spread more widely. According to the role of the pion field in the $\chi \mathrm{QSM}$, where one can associate the contribution of the discrete level to the quark core and the contribution of the negative continuum states to a pion cloud, this means that the range of the pion cloud increases and the nucleon becomes larger. Actually, the mean square radius $\left\langle r_{E}^{2}\right\rangle$ increases from $0.67 \mathrm{fm}^{2}$ in the case of the physical pion to $0.79 \mathrm{fm}^{2}$ in the chiral limit. With increasing pion mass up to $1.2 \mathrm{GeV}$ this trend is confirmed with the nucleon becoming smaller and smaller [36].

The normalized radial distribution of angular momentum $4 \pi r^{2} \rho_{J}(r) / J_{N}$, is shown in Fig. $2 b$ as a function of $r$ for $m_{\pi}=0$ and $140 \mathrm{MeV}$. For any $m_{\pi}$ at small $r$ one finds $\rho_{J}(r) \propto r^{2}$. The mean square radius $\left\langle r_{J}^{2}\right\rangle$ decreases with increasing $m_{\pi}$ [36] in agreement with the idea of a shrinking pion cloud. For a physical pion one finds $\left\langle r_{J}^{2}\right\rangle=1.32 \mathrm{fm}^{2}$. At large $r$ in the chiral limit $\rho_{J}(r) \propto 1 / r^{4}$ such that $\left\langle r_{J}^{2}\right\rangle$ diverges.

Fig. 3a shows the pressure $p(r)$ as function of $r$. In the physical situation $p(r)$ takes its global maximum at $r=0$ with $p(0)=0.23 \mathrm{GeV} / \mathrm{fm}^{3}=3.7 \cdot 10^{34} \mathrm{~Pa}$. This is $\mathscr{O}(10-100)$ higher than the pressure inside a neutron star. Then $p(r)$ decreases monotonically (becoming zero at $r_{0}=$ $0.57 \mathrm{fm}$ ) till reaching its global minimum at $r_{p, \min }=0.72 \mathrm{fm}$, after which it increases monotonically remaining, however, always negative. The positive sign of the pressure for $r<r_{0}$ corresponds to the repulsion among quarks imposed by Pauli principle, while the negative sign in the region $r>r_{0}$ means attraction in agreement with the idea of a pion cloud responsible for binding the quarks to form the nucleon. The subtle balance between repulsion and attraction, ultimately producing a stable soliton, can be better appreciated from Fig. 3b showing $r^{2} p(r)$, where the shaded regions have the same surface areas but opposite sign and cancel each other within numerical accuracy.

\section{References}

[1] K. Goeke, M.V. Polyakov, and M. Vanderhaeghen, Prog. Part. Nucl. Phys. 47, 401 (2001).

[2] M. Diehl, Phys. Rep. 388, 41 (2003).

[3] X. Ji, Ann. Rev. Nucl. Part. Sci. 54, 413 (2004). 
[4] A.V. Belitsky, and A.V. Radyushkin, Phys. Rep. 418, 1 (2005).

[5] S. Boffi, and B. Pasquini, Riv. Nuovo Cim. 30, 387 (2007).

[6] F.M. Dittes, D. Müller, D. Robaschik, B. Geyer, and J. Hořejši, Phys. Lett. B209, 325 (1988).

[7] D. Müller, D. Robaschik, B. Geyer, F.M. Dittes, and J. Hořejši, Fortschr. Phys. 42, 101 (1994).

[8] X. Ji, Phys. Rev. Lett. 78, 610 (1997).

[9] A.V. Radyushkin, Phys. Lett. B380, 417 (1996).

[10] X. Ji, Phys. Rev. D55, 7114 (1997).

[11] A.V. Radyushkin, Phys. Lett. B385, 333 (1996).

[12] M. Burkardt, Phys. Rev. D62, 071503 (2000); (E) ibitem, 119903 (2002).

[13] M. Burkardt, Phys. Rev. D72, 094020 (2005).

[14] M.V. Polyakov, Phys. Lett. B555, 57 (2003).

[15] Ph. Hägler, et al. (The LHPC and MILC Collaborations). Phys. Rev. D77, 094502 (2008).

[16] M. Göckeler, et al. (QCDSF Collaboration), Phys. Rev. Lett. 92, 042002 (2004).

[17] M. Dorati, T.A. Gail, and T.R. Hemmert, Nucl. Phys. A798, 96 (2008); PoS. Lat2007, 071 (2007).

[18] D. Brömmel, et al. (QCDSF-UKQCD Collaboration), PoS. LAT2007, 364 (2007).

[19] A. Airapetian et al. (Hermes Collaboration), JHEP 0806:066, 2008.

[20] M. Mazouz et al. (JLab Hall A Collaboration), Phys. Rev. Lett. 99, 242501 (2007).

[21] M. Vanderhaeghen, P.A.M. Guichon, and M. Guidal, Phys. Rev. D60, 094017 (1999).

[22] V. Guzey, T. Teckentrup, Phys. Rev. D74, 054027 (2006).

[23] B. Pasquini, and S. Boffi, Phys. Rev. D76, 074011 (2007).

[24] S. Boffi, B. Pasquini, and M. Traini, Nucl. Phys. B649, 243 (2003); Nucl. Phys. B 680, 147 (2004); B. Pasquini, M. Pincetti, and S. Boffi, Phys. Rev. D72, 094029 (2005).

[25] B. Pasquini, and S. Boffi, Phys. Rev. D73, 094001 (2006); B. Pasquini, M. Traini, and S. Boffi, Phys. Rev. D71, 034022 (2005).

[26] B. Pasquini, S. Cazzaniga, and S. Boffi, Phys. Rev. D78, 034025 (2008); B. Pasquini, arXiv:0807.2825 [hep-ph].

[27] M. Burkardt, Int. J. Mod. Phys. A18, 173 (2003).

[28] M. Diehl, and Ph. Hägler, Eur. J. Phys. C44, 87 (2005).

[29] M. Göckeler, et al. (QCDSF-UKQCD Collaboration), Phys. Rev. Lett. 98, 222001 (2007).

[30] B. Pasquini, and S. Boffi, Phys. Lett. B653, 23 (2007).

[31] M. Burkardt, Phys. Rev. D66, 114005 (2002); Nucl. Phys. A735, 185 (2004).

[32] A. Airapetian et al. (Hermes Collaboration), Phys. Rev. Lett. 94, 012002 (2005).

[33] M. Alekseev, et al. (COMPASS Collaboration), arXiv:0802.3023 [hep-ex].

[34] M. Diehl, A. Manashov and A. Schäfer, Phys. Lett. B622, 69 (2005); Eur. Phys. J. A31, 335 (2007).

[35] C. Cebulla, K. Goeke, J. Ossmann and P. Schweitzer, Nucl. Phys. A794, 87 (2007).

[36] K. Goeke, et al., Phys. Rev. D75, 094021 (2007).

[37] M. Wakamatsu, and Y. Nakakoji, Phys. Rev. D74, 054006 (2006). 